\title{
Resources Management of High Speed Downlink Packet Access Network in the Presence of Mobility
}

\author{
Abdulaleem Ali Almazroi ${ }^{1}$ \\ ${ }^{1}$ Department of Computer Science \\ Rafha Community College \\ Northern Border University, Arar, 91431, Saudi Arabia
}

\begin{abstract}
High-Speed Downlink Protocol Access (HSDPA) is a mobile telephony protocol. It is designed to increase data capacity and transfer rate. This paper presents a resource allocation strategy in the HSDPA broadband network. An admission check is proposed. It divides the coverage area of a base station (Node-B) into several regions based on the principle of Adaptive Modulation and Coding (AMC) efficiency.

The call admission control (CAC) mechanism distinguishes two RT and NRT traffic according to the type of service requested by the user. It dynamically allocates an effective bandwidth to each accepted call in the system based on its modulation efficiency and maintains its initial rate during its communication.
\end{abstract}

Keywords-HSDPA Network; Admission Control; Performance Evaluation; Mobility

\section{INTRODUCTION}

To know the coverage and quality of the mobile network, price is not the only element that must be taken into account. We should also look at the coverage and quality of the operator's network. Several factors can influence the reception quality of the mobile network, such as physical barriers, the distance from the user to the relay antenna and the number of people connected at the same time [1].

In order to provide the quality of service required and compare services, new mechanisms are needed. These mechanisms include resource reservation protocols, packet scheduling policies and admission controls. CDMA (Code Division Multiple Access) is the most advanced technique of multiplexing, intended to be used especially on third generation mobile telephony networks such as UMTS [2]. It is an access method where all users share the same frequency band simultaneously and all the time. The spread spectrum technique is used to assign to each user a unique code or sequence that determines the frequencies and power used. The signal containing the information of the transmitter is modulated with the sequence assigned to it, and then the receiver searches for the sequence in question. By isolating all sequences from other users (which appear as noise), the original signal of the user can then be extracted [3].

There are nevertheless different CDMA variants. The Wideband-Code Division Multiple Access (WCDMA) protocol is based mainly on the DS-CDMA (Direct SequenceCDMA), direct sequence spectrum spreading process [4]. In this type of spread spectrum, the information signal is directly modulated by a sequence or code called "spreading code". This is the same technique as CDMA, using $5 \mathrm{MHz}$ channels in UMTS.

A WCDMA software extension called High-Speed Downlink Packet Access (HSDPA) was introduced to improve the rate of downlink where more information will be transported. With the HSDPA technique the throughput can reach $14 \mathrm{Mbps}$ in the $3.5 \mathrm{G}$ network or $\mathrm{HSP}+$ [5].

In this paper, we are interested in the study of quality (QoS) of service in the HSDPA broadband network extension of the UMTS-WCDMA network. The QoS depends directly on the quality of the radio channel associated with the mobile and varies according to its state (good or bad). This quality of service becomes too complicated to guarantee and varies according to the level of low or high mobility, and thus, depending on the type of intra or inter-cell mobility of the user. The HSDPA cell is decomposed into a finite number of regions of the same centre and each of them is associated with a given modulation (mean state of the transmission channel).

We explicitly calculate the rate of migration of calls migrating from one region to another according to the estimated average number of active calls in this region and the probability of migration. In addition, the new mechanism will dynamically affect the bandwidth necessary to maintain the initial rate of a call (real or non-real time) regardless of its position in the cell. This mechanism gives more priority to migrating calls by reserving bandwidth only for them. The value of this band can be controlled by the operator according to the periods of mobility (strong or weak) of the network.

The remainder of this paper is divided into six sections. After introducing, the HSDPA technique is presented in Section 2. Section 3 describes related work on mobility management and admission control mechanism for WCDMA and HSDPA systems. Section 4 presents a model of an HSDPA cell. In section 5, experiments and results are detailed. Finally, conclusions are presented in Sections 6.

\section{High SPEed Downlink PACKet Access (HSDPA)}

To provide UMTS high bandwidth interactive, streaming and background services greater than 2 Mbps, 3GPP defined HSDPA (High Speed Downlink Packet Access) in Release 5 [1]. While the maximum throughput allowed on a UMTS link is $2 \mathrm{Mbps}$ for a bandwidth of $5 \mathrm{MHz}$, the HSDPA, thanks to its 16-QAM modulation, allows 10 Mbps. Thus, thanks to the introduction of the new 64-QAM modulation in Release 7, the 
theoretical rate of $14 \mathrm{Mbps}$ is possible via the HSDPA network [2].

The HSDPA technology is the software evolution of the WCDMA technology of the Release 99. It is equipped with a set of properties, the combination of which increases the data capacity as well as the transfer rate up to more Of $10 \mathrm{Mbps}$. Figure 1 shows system architecture with HSDPA. Among these properties, we find known techniques used in evolutionary standards such as the GSM/EDGE and defined by the following points:

- The AMC adaptive modulation and coding technique allows adaptive transmission rate variation to compensate for signal degradation due to propagation conditions. However, the performance of this technique is quite sensitive to errors in the estimation of channel conditions and implicit delays in its transmission to Node-B [31].

- A fast hybrid retransmission method called Hybrid Automatic Repeat reQuest (H-ARQ): This method is seen as a complement to the previous AMC by providing the possibility of adjusting the transmission rate more finely. The Node-B transmits a data packet to the mobile. If after a certain time the latter does not send a positive acknowledgment (ACK, Acknowledgment) or if the acknowledgment is negative (NACK, Negative-Acknowledgment) then, the Node-B considers that the packet has not received and returns the same package again. The mobile keeps it and combines it with the packets retransmitted subsequently. This type of retransmission is called soft combining and there is another type called Incremental Redundancy [6]. This increases the probability of correctly decoding the information.

- Fast packet scheduling algorithms: The two previous techniques make it possible to improve the performance of the radio link by changing the transmission rate according to the instantaneous characteristics of the channel. The scheduling algorithms allow selecting the cell users to whom the High Speed-Downlink Shared Channel data channel (HS-DSCH) must be allocated during an Interval Time Transmission (ITT) transmission time interval. Among the strategies for allocating radio resources called scheduling are Max C/I, PF (Proportional Fair) and FFTH (Fast Fair Throughput) [7].

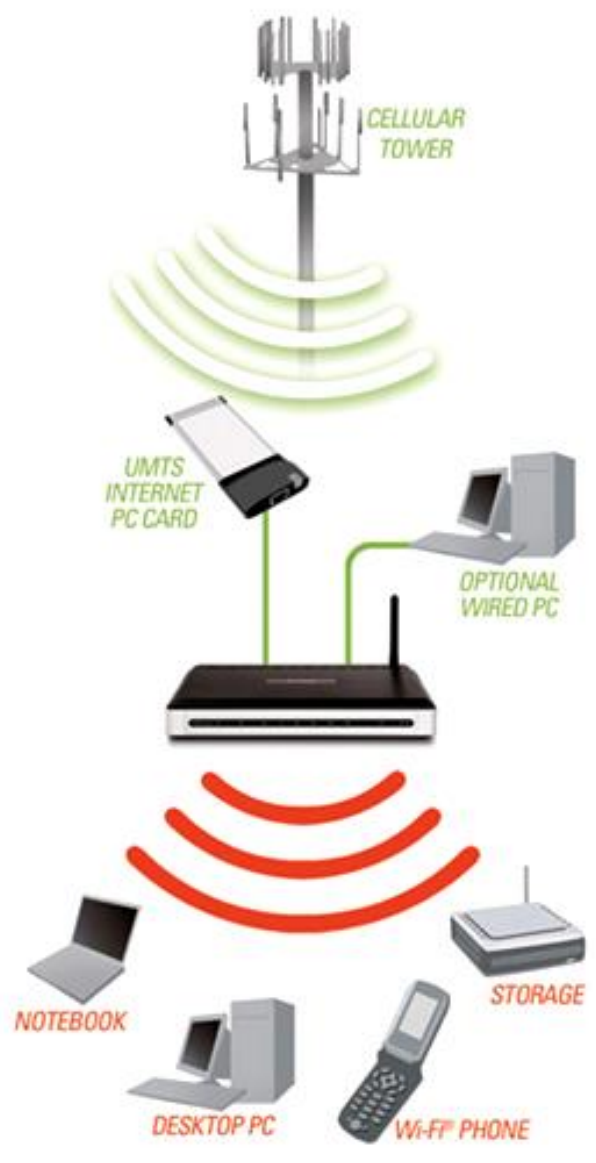

Fig. 1. System architecture with HSDPA.

\section{RELATED WORKS}

This section describes the literature already existing in which this work derives its motivations. It deals with work, different models of performance, capacity calculation, mobility management and admission control mechanism for WCDMA and HSDPA systems.

Numerous works have been developed in the literature to study the capacity of wireless networks. In [8], Zhang et al. presents a method for calculating the uplink capacity of the WCDMA system uplink [9]. The authors consider various factors, such as noise ratio, interference level and power control errors. This method calculates the capacity utilisation of the UMTS/WCDMA system on the basis of the probability of outage. 
Moreover, in the work developed in [10], the capacity of the uplink is studied with two traffics: RT call is transmitted continuously, and NRT is transmitted in time-sharing. In [11], the author calculates the upstream link capacity of CDMA systems with an idealised power control that contains besteffort applications, i.e. applications whose transmission rate can be controlled. The author allows a variety of services and therefore mobile terminals may have different qualities of services depending on the type of call. It guarantees the quality of service of calls in progress assuming that there is an access control exercised in order to prevent a new call from arriving when the system is saturated.

The authors in [12] consider 2 traffics, namely, RT traffic and best effort. They then study the impact of best effort fixed bandwidth on the system's Erlang capacity and show that a very low bandwidth reserved for best effort calls indicates very long call duration.

In [13], they assume that RT calls have resources dedicated by the system, whereas NRT calls share free resources. They investigate the probability of blocking the new RT traffic calls and the expected residence time for NRT traffic calls on the two uplink and downlink transmission links with and without macro diversity. Then they propose a CAC (call admission control) in order to have a QoS for both traffics and give an extension of their work including the handover.

In [14], a comparison was made with the maximum capacity obtained by the theory of information. The model describes a relationship between the data transmission rates and the amount of resources used in terms of power level in the above-mentioned systems. The authors present results that emphasise the importance of modelling the system by taking into account the arrival and the dynamic departure of calls.

The authors in [15] define a new method by extending the classical Kaufman-Roberts formula in the CDMA system supporting best effort services. The services of this class can dynamically adapt their transmission rate according to interference. The authors use the Kaufman-Roberts formula to calculate steady state by setting transmission rates in the system. They give an approximation of a Markov chain irreversible by the reversible Markov chain and obtain lower and upper limits of probabilities of blocking of the new calls in the state of equilibrium.

A new call is accepted by the CAC if it does not degrade the QoS of ongoing calls [16], [17]. The CAC in the thirdgeneration UMTS network has also been the subject of several researches $[18,19,20,21]$ for the uplink.

In [22], the authors present a model for studying the HSPA system's ability by combining the two uplink and downlink links with the presence of two classes of RT and best effort service. The best effort calls stay in the system for a long time if there are fewer free resources and leave quickly when they have more free resources. The authors analyse the
HDR/HSDPA system by offering system performance and offer an admission control for best effort calls in both transmission links.

In [23], Oleksandr presents the HS-SFN, a multipoint transmission schemes for HSDPA, which can increase the performance of HS-SFN. The author considers the HS-SFN by studying the radio resource management algorithms for HSDPA radio access network. The researchers in [24], have studied the 3 UK's HSDPA network performance. The Iub backhaul capacity limitations have been analysed using Maidenhead HSDPA network example.

The admission control is based on the sub-division of the cell into a finite number of regions and each region has a different modulation. It allows guaranteeing to each mobile its initial rate whatever its position in the cell or when the quality of its radio link becomes bad. The decomposition of the cell into regions has interested several researchers [25, 26, 27, 28].

\section{Modelisation Of AN HSDPA Cell}

To better manage the bandwidth of the system, it is necessary to take into consideration the variation of the channel state. Users nearly to the station generally perceive a good quality of the channel and therefore a satisfactory quality of service. On the other hand, those who are far away, their quality of service have been degraded. We studied in this work the variation of the channel state using the HSDPA technique; WCDMA system software extension. Indeed, in the high-speed HSDPA network, the system dynamically adapts the quality of service according to the state of the channel perceived by the user. However, when the quality of the channel changes from good to bad, the user is at a degraded quality of service. We use AMC (Adaptive Modulation and Coding). This makes it possible to maintain a constant flow rate to the user regardless of his position and taking into account his mobility [29].

The transmitted signal is modified taking into consideration the quality of the signal through a process known as adaptation to the radio link, or AMC (Adaptive Modulation and Coding). The Adaptive Modulation and Coding becomes a standard approach in high-speed networks like High Speed Downlink Packet Access [31, 32, 33]. As mentioned in [34], the major idea of AMC is the dynamic change of coding and modulation coding depending on the conditions of the radio channel.

The cell is divided into $r$ regions of radius $R_{j}$. The area of the $j$-th region is $S_{j}$. Figure 2 shows how the HSDPA cell is divided into several modulation regions with AMC. We consider that the real-time (RT) and non-real-time (NRT) call arrival processes are independent. As well, service times are independent and exponentially distributed. $\lambda_{0, c}^{j}$ is the arrival rate of the new calls of class- $c$ in region $j .1 / \mu_{R T}$ is the average duration of a real-time call. The NRT call duration depends on the average file size to be downloaded in E (Pay) bits [35], given by:

$$
\frac{1}{\mu_{N R T}}=\frac{E(\text { Pay })}{R_{N R T}}
$$




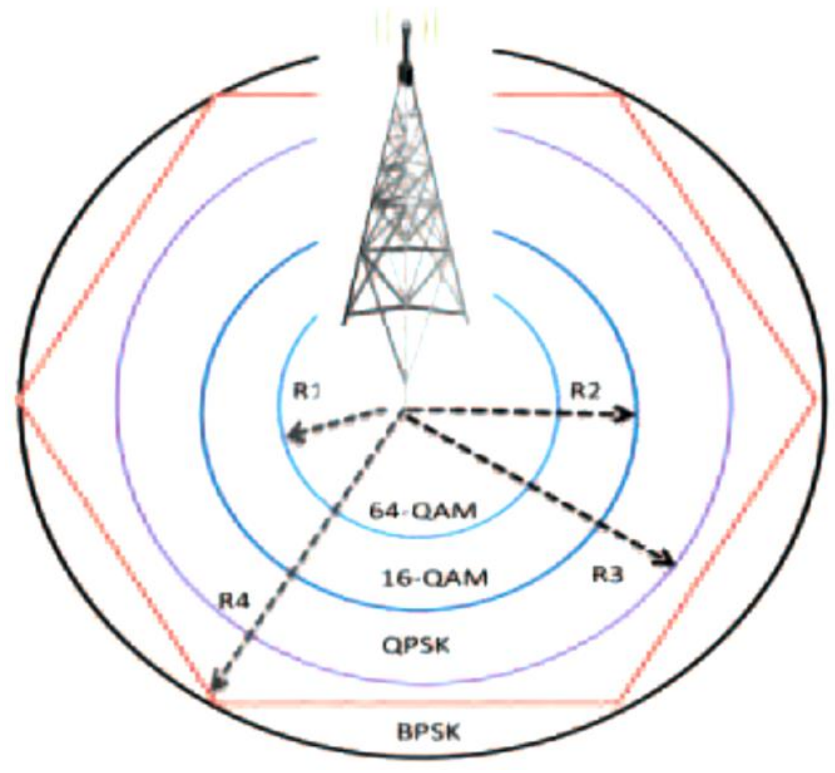

Fig. 2. The HSDPA cell divided into several regions with AMC.

We recall that the cell is decomposed into $r$ regions and in each of them we have two different pass bands $\Delta_{R T}^{i}$ and $\Delta_{R T}^{i}$. This shows that there are $2 r$ call classes in the system: $r$ realtime call classes and $r$ non-real-time call classes. When a user changes his line to another before he finishes his service, we are talking about intra-cell mobility (between regions within the same cell).

\section{EXPERIMENTS AND RESULTS}

Some numerical results based solely on the path loss according to the distance between the Node-B and the mobile, are presented here. The HSDPA central cell is sub-divided into three regions with modulations: QPSK 1/2, 16-QAM 3/4 and 64QAM 3/4. The energy transmitted from a class- $c$ bit by noise $E_{c} / N_{0}$ is $3.4 \mathrm{~dB}$ for real-time calls and equal to $2.7 \mathrm{~dB}$ for nonreal-time calls. The constant bit rate for RT calls is $R_{R T}=0.3$ Mbps and that of NRT calls is $R_{N R T}=0.15 \mathrm{Mbps}$. In addition, we study the impact of low user mobility between regions. Users can move between adjacent regions and the average time in the region is $300 \mathrm{~s}$.

\section{A. Impact of the scenario without intra mobility:}

The scenario without intra mobility is illustrated in Figures 3 and 4. These two figures respectively present the probabilities of blocking RT and NRT calls according to the real-time call arrival rate for different modulation efficiencies. In both figures we remark that the probability of call blocking in region three where the modulation efficiency is QPSK is greater than those of the anterior regions (i.e. the 64-QAM and two 16-QAM regions). This is due to the fact that a user in region three requires more bandwidth than in the other regions for both traffics, i.e.

$$
\Delta_{R T}^{1}<\Delta_{R T}^{2}<\Delta_{R T}^{3} \text { and } \Delta_{N R T}^{1}<\Delta_{N R T}^{2}<\Delta_{N R T}^{3} .
$$

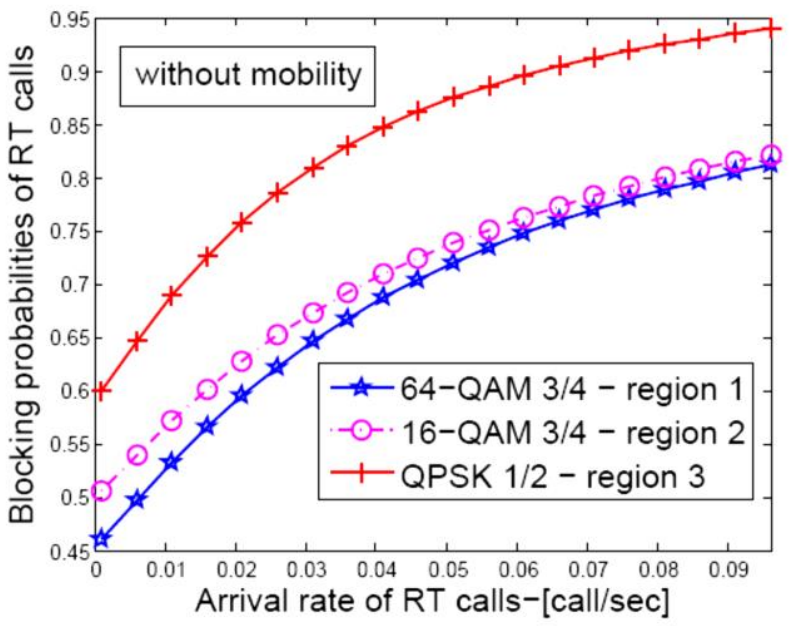

Fig. 3. Probabilities of real-time call blocking according to RT call arrival rate.

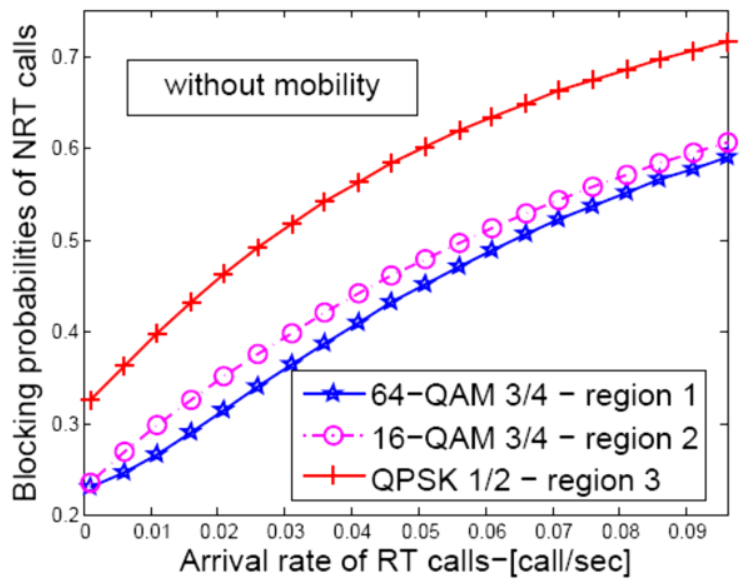

Fig. 4. Probabilities of real-time call blocking according to the NRT call arrival rate.

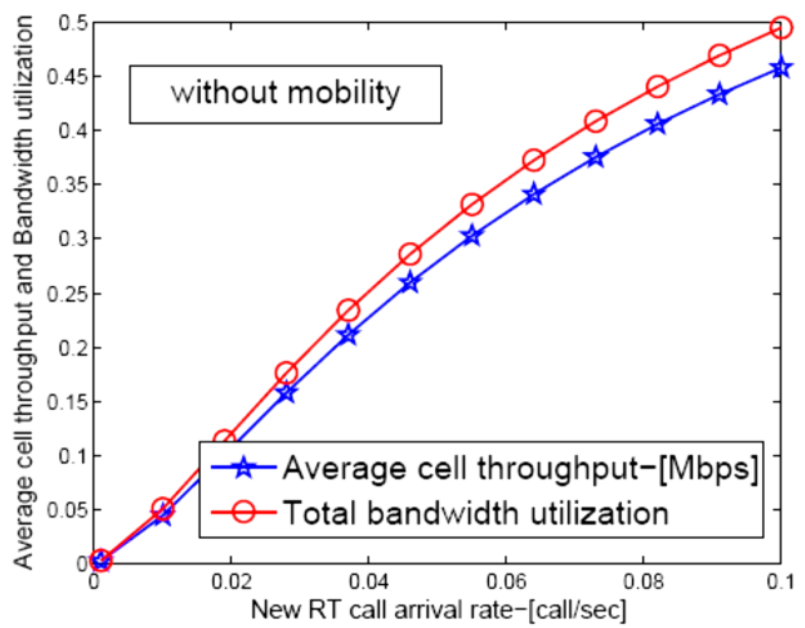

Fig. 5. Total bandwidth and bandwidth usage based on RT call arrival rate. 
Moreover, the main difference between the probability of RT and NRT call blocking classes is that there is more blocking for the RT calls. Because RT calls require more bandwidth in our digital environment than is required by NRT calls in the same region. These results are due to the fact that our resource allocation strategy gives more priority to calls coming from regions close to the base station than those in regions with low modulation, while maintaining the same throughput for all these calls. Total bandwidth and throughput utilisation are illustrated in Figure 5. As soon as a call is accepted by the CAC mechanism, the system must maintain a constant flow during its service. This implies that the total throughput of the system increases, and also that the total utilisation of the bandwidth increases.

\section{B. Impact of the mobility scenario:}

Let us now focus on the impact of the intra- and inter-cell mobility scenario on HSDPA performance. The probabilities of real-time and non-real-time call blocking based on bandwidth reserved for migrating calls is illustrated in Figures 6 and 7. This probability is improved with the improvement in this proportion; due to our CAC, strategy favours the mobility of newcomers by this proportion of resources.

The probabilities of RT and NRT call loss due to bandwidth reserved for migrating calls is represented in the logarithmic scale in Figure 8. The loss of current calls occurs when they start from the high-modulation region (fewer resources per call) to the low-modulation region (more resources per call).

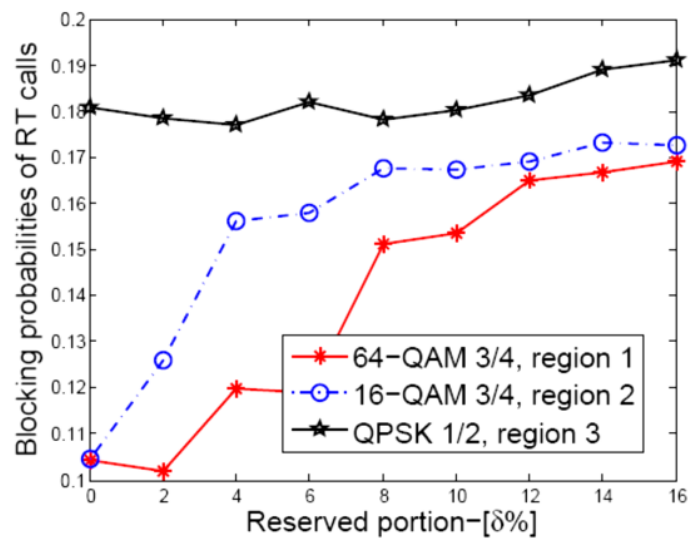

Fig. 6. Probabilities of real-time call blocking based on bandwidth reserved for migrating calls.

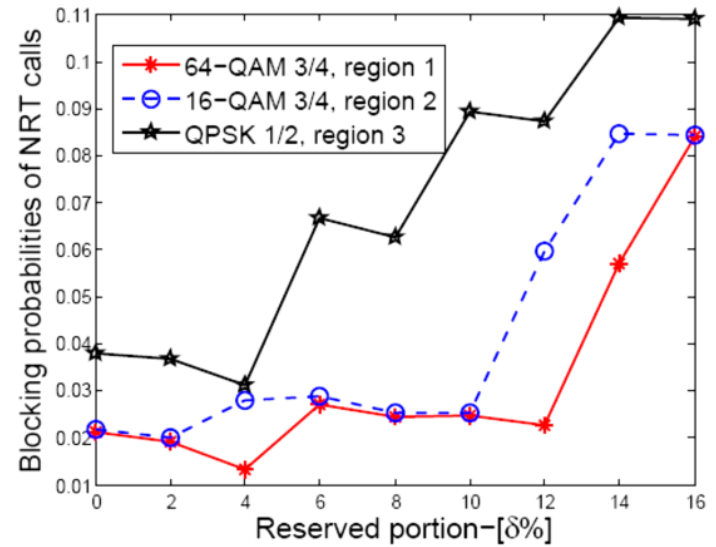

Fig. 7. Probabilities of non-real-time call blocking based on bandwidth reserved for migrating calls.

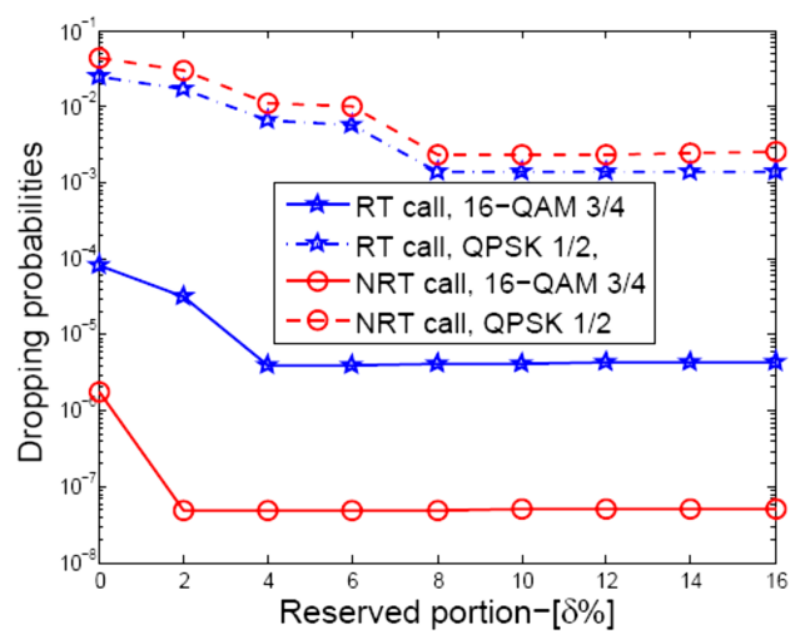

Fig. 8. Probabilities of RT and NRT call loss due to bandwidth reserved for migrating calls.

Figure 9 represents the probability of loss based on bandwidth reserved for migrating calls. The increase of the interference factor implies a decrease of the total flow and therefore a reduction in the space of call states. In addition, fewer calls will occupy the entire bandwidth of the system when inter-cell interference is considered. This degradation in the capacity of the HSDPA system confirms the result obtained in [36]. 
We now assume that the service provider reserves $\theta_{m}=8 \%$ of total bandwidth for mobile call management. In addition, Figure 10 represents the total utilisation of the bandwidth occupied by the RT/NRT calls. In this figure, one notices the direct impact of the $F$-factor on the way the calls use the bandwidth. For an arrival rate equal to $0.04 \mathrm{calls} / \mathrm{s}$, it can be seen that $55 \%$ of the bandwidth is used when inter-cell interference is high $F$-factor $=0.15$. On the other hand, $73 \%$ is occupied when the interference is weak F-factor $=0.05$, which exploits the capacity of the system by the current calls. In addition, Figure 11 compares the probabilities of losses for the calls in progress RT and NRT. The results are illustrated based on the arrival rate of new RT calls. The main point is that our CAC mechanism capable of keeping a low probability of loss for RT and NRT calls if there is less inter-cell interference.

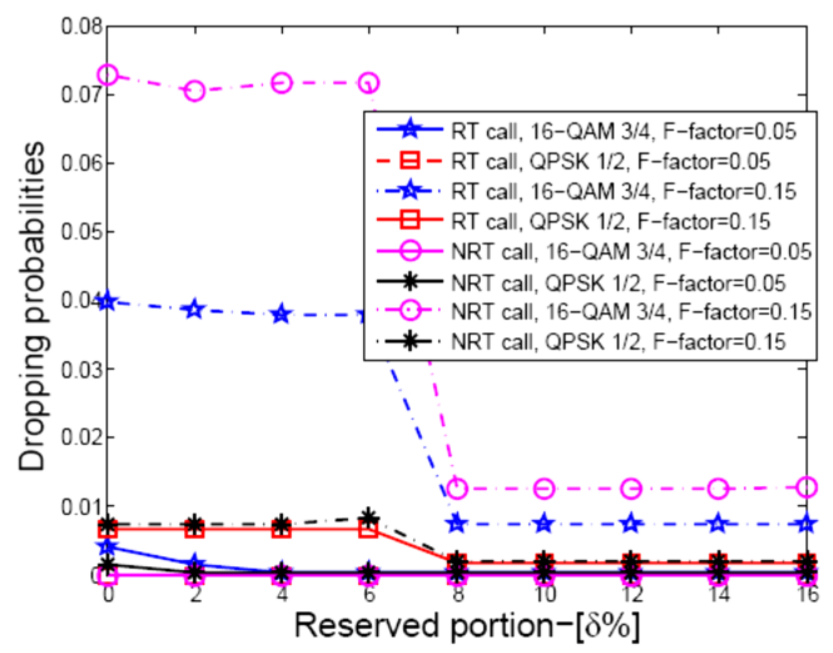

Fig. 9. Probability of loss based on bandwidth reserved for migrating calls.

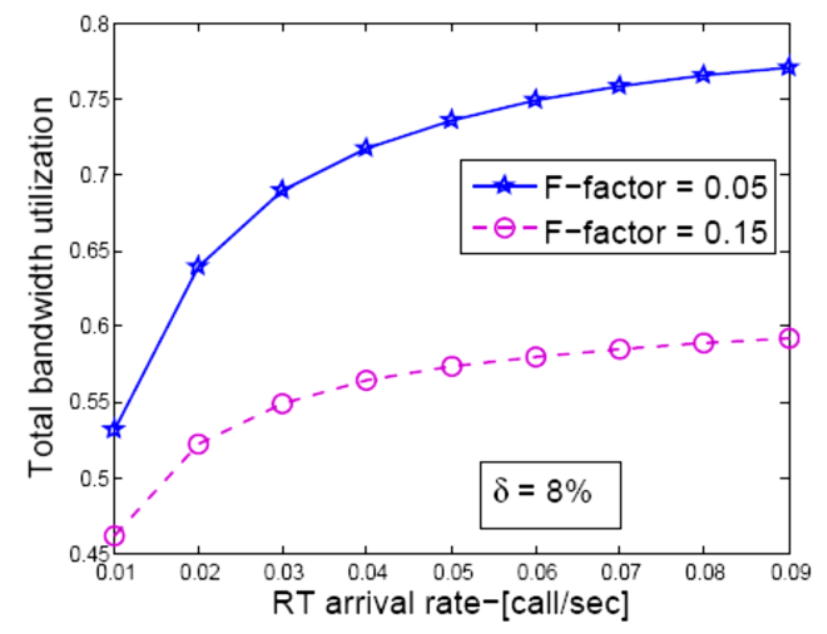

Fig. 10. Total bandwidth usage based on real-time call arrival rate.

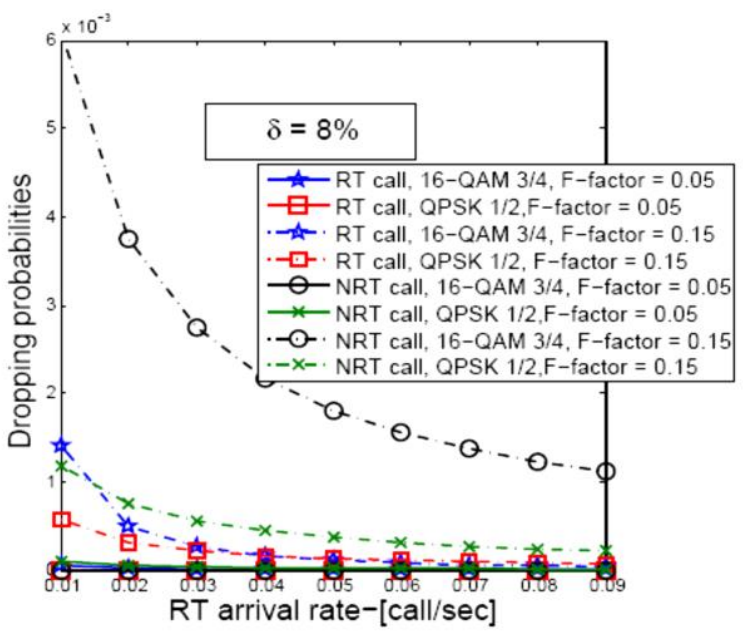

Fig. 11. Probability of loss based on real-time call arrival rate.

\section{CONCLUSIONS}

In this paper we focused on analysing the HSDPA system as an extension of the 3G UMTS network. In the HSDPA broadband network, the modulation efficiency generally changes as a function of channel quality. In other words, good signal quality allows the user to be assigned as a high modulation with several bits per symbol and vice versa. However, when the modulation changes from high to low, the user is in a degraded rate.

This problem is dealt with in this paper by the adaptation of the bandwidth when the modulation changes and in order to maintain a constant rate of the user independently of its position. The reservation of resources depends on what a service provider wants to do based on the traffic (minimise the probabilities of loss of calls during communication and the blocking of new calls). The service provider may change the value reserved for user mobility in periods of high or low mobility. We have shown that inter-cell mobility and inter-cell interference forces our CAC mechanism to allocate more bandwidth to the mobiles to achieve a constant rate. This reduces the space of possible system states and therefore, less total system throughput.

\section{REFERENCES}

[1] T. Nishith, H.R. Jeffrey. Cellular Communications: A Comprehensive and Practical Guide. John Wiley \& Sons, Sep 12, 2014.

[2] C. Hyun-Dong, K. Hyunjung, S. Seung-Joon. Flow based 3G/WLAN vertical handover scheme using MIH model. In Proc 2013 International Conference on Information Networking (ICOIN), pp: 658-663, 2013.

[3] Y. Donoso, C. Lozano-Garzon, M. Camelo, P. Vila. A Fairness Load Balancing Algorithm in Heterogeneous Wireless Networks using a Multihoming Strategy. International Journal of Computers, Communications \& Control, Vol.9 (5),pp:555-569, 2014.

[4] Khosrow-Pour, Mehdi. Inventive Approaches for Technology Integration and Information Resources Management. IGI Global, 30 juin 2014. 
[5] Antonio Capone. SMARTPHONES ANALYSIS - ESTIMATING RNC UNIT LOAD. PhD Thesis, University POLITECNICO DI MILANO. 2012.

[6] T. Mamadou, S. Javier. UMTS (réseaux et télécommunications, 2nd edition), Hermes - Science, 2004.

[7] P. Ameigeiras, J. Wigard, and P. Mogensen. Performance of packet scheduling methods with different degree of fairness in HSDPA. In Proc of Vehicular Technology IEEE Conference, Vol. 2, pp: 860-864, 2004.

[8] Q. Zhang and O. Yue. UMTS air inteface voice/data capacity part 1: reverse link analysis. In Proc of IEEE Veh. Technol. Conf, pp: 2725$2729,2001$.

[9] A. J. Viterbi. CDMA Priciples of Spread Spectrum Communication. Addition-Wesley, 1995.

[10] N. Mandayam, S. Barberis, and J. Holtzman . Performance and capacity of a voice/data CDMA system with variable bit rate sources. Journal of Mobile Multimedia Communications, Academic Press Inc.1997.

[11] E. Altman. Capacity of multi-service CDMA cellular networks with best-effort applications. In Proc of MOBICOM, Atlanta, Georgia, USA, 2002.

[12] N. Hegde and E. Altman. Capacity of multiservice WCDMA networks with variable gos. In Proc of IEEE Wireless Communications and Networking Conference (WCNC), 2003.

[13] J. M. Kelif and E. Altman. admission and gos control in multiservice WCDMA system. In Proc of ECUMN'2004, Porto, Portugal, 2004.

[14] T. Bonald and A. Proutiére. On the Traffic Capacity of Cellular Data Networks. In Proc of International Teletraffic Congress, 2005.

[15] G. Fodor and M. Telek. Blocking Probability Bounds in Multi-service CDMA Networks. In Proc of International Teletraffic Congress, Beijing, China, 2005.

[16] X. Tang and A. Goldsmith. Admission control and adaptive CDMA for integrated voice and data systems. In Proc of IEEE VTC, pp: 506-510, Rhodes, Greece, 2001.

[17] C. W. Leong and W. Zhuang. Call admission control for voice and data traffic in wireless communications. Journal of Computer Communications, Vol 25 (10), pp: 972-979, 2002.

[18] S. E. Elayoubi, T. Chahed, M. Tlais, and A. Samhat. Measurementbased admission control in UMTS. Annals of Telecommunications on Traffic Engineering and Routing, 2004.

[19] S. E. Elayoubi, T. Chahed, and L. Salahaldin. Optimization of radio resource management in UMTS using pricing. Journal of Computer Communications, Vol 28(15), 2005.

[20] E. Elayoubi, T. Chahed, and G. Habuterne. Mobility-aware admission control schemes in the downlink of third generation wireless systems. IEEE Transactions on Vehicular Technology, 2006.

[21] M. Assaad. Cross Layer Study in HSDPA System. PhD Thesis, France, 2006.

[22] Puchko Oleksandr. Multipoint Transmission Scheme for HSDPA. PhD Thesis, University of Jyväskylä, 2013.
[23] F. Hayder, E. Hepsayader, N. Piniccu. Performance Analysis of a Live Mobile Broadband-HSDPA Network. In Proc of the 73rd IEEE Vehicular Technology Conference, VTC Spring 2011, 15-18 May 2011, Budapest, Hungary, 2011.

[24] Y, Souleimen, A, Kaled. An Enhanced Buffer Management Scheme for Multimedia Traffic in HSDPA. In Proc of Conference on Next Generation Mobile Applications, Services and Technologies (NGMAST), pp: 292-297, 2016.

[25] J. Li and S. Sampalli. Cell mobility based admission control for wireless networks with link adaptation. In Proc of ICC, 2007.

[26] F. Baskett, K. Chandy, R. Muntz, and F. Palaclos. Open, closed and mixed networks of queues with different classes of customers. Journal of the ACM (JACM), Vol. 22(2), pp: 248-260, 1975.

[27] X. Chao, M. Miyazawa, M. Pinedo, and B. Atkinson. Queuing networks : Customers, signals and product form solutions. Journal of the Operational Research Society, Vol. 52 (5), pp: 600-601, 2001.

[28] T. Bonald and A. Proutière. Wireless downlink data channels: user performance and cell dimensioning. In Proc of the 9th annual international conference on Mobile computing and networking, New York, NY, USA, pp: 339-352, 2003.

[29] R. Yang, Y. Chang, J. Sun, D. Yang. Traffic Split Scheme Based on Common Radio Resource Management in an Integrated LTE and HSDPA Networks.In Proc of 2012 IEEE Vehicular Technology Conference (VTC Fall), pp: 1-5, 2012.

[30] Y. Jin, B. Liu, L. Qiu,J. Xu,Y.Huang. QoS aware energy efficient resource allocation in HSDPA systems. In Proc of 2014 IEEE Wireless Communications and Networking Conference (WCNC), pp: 1643 - 1648, 2014

[31] R. Kwan, P.Chong, and M. Rinne. Analysis of the adaptive modulation and coding algorithm with the multicode transmission. Journal of Vehicular Technology, Vol. 4, pp: 2007-2011, 2002.

[32] M.Nakamura, Y. Awad, and S. Vadgama. Adaptive control of link adaptation for high speed downlink packet access (hsdpa) in w-cdma. In Proc of 5th International Symposium on Wireless Personal Multimedia Communications, Vol. 2, pp: 382-386, 2002.

[33] R. Qiu, W. Zhu, and Y. Zhang. Third-generation and beyond (3.5g) wireless networks and its applications. In Proc of IEEE International Symposium, Vol. 1, pp: 41-44, 2002.

[34] A. B. Downey. The structural cause of file size distributions. SIGMETRICS Performance Evaluation Review, Vol. 29(1), pp: 328329, 2001.

[35] T. Chahed, E. Altman, and S. Elayoubi. Joint uplink and downlink admission control to both streaming and elastic flows in CDMA/HSDPA systems. Journal of Performance Evaluation, Vol 65(11-12), pp: 869882, 2008.

[36] A. Viterbi, A. Viterbi, and E. Zehavi. Other-cell interference in cellular power-controlled CDMA. IEEE Transactions on Communications, Vol.42, pp: 1501-1504, 1994. 